\title{
GERMINAÇÃO DE SEMENTES DE Mentha spp. EM FUNÇÃO DO COMPRIMENTO DE ONDA DE LUZ
}

Germination of seeds of Mentha spp. according to the light wave length

Germinación de semillas de Mentha spp. según la longitud de onda ligera

\section{Kaique Aguiar Silva ${ }^{1}$, Weslany Silva Rocha*2, Sara Monteiro Pinto ${ }^{2}$,} Ildon Rodrigues do Nascimento ${ }^{3}$, Valéria Gomes Momenté

${ }^{1}$ Aluno de graduação, Engenharia de Alimentos, Universidade Federal do Tocantins, Palmas, Brasil.

${ }^{2}$ Técnica do Laboratório de Fitoterapia, Engenharia de Alimentos, Universidade Federal do Tocantins, Palmas, Brasil.

${ }^{3}$ Laboratório de Olericultura, Agronomia, Universidade Federal do Tocantins, Gurupi, Brasil.

${ }^{4}$ Coordenadora do Laboratório de Fitoterapia, Engenharia de Alimentos, Universidade Federal do Tocantins, Palmas, Brasil.

*Correspondência: Laboratório de Fitoterapia, Universidade Federal do Tocantins, Av. NS 15, 109 Norte, Palmas, Tocantins, Brasil.CEP:77.010-090.e-mail weslany.rocha@mail.uft.edu.br.

\section{RESUMO}

Hortelã spp. é uma planta medicinal herbácea, propagada por sementes ou por estacas, que apresenta grande importância como antimicrobiana e anti-inflamatória em estudos etnobotânicos e etnofarmacológicos. Importância esta, devido à presença de um dos seus principais constituintes químicos, o mentol, encontrado principalmente nas folhas, muito utilizado para fins medicinais, alimentícios e cosméticos. Com isto, objetivou-se, avaliar a influência do comprimento de ondas de luz na germinação de sementes de Mentha spp. Para isto, empregou-se delineamento experimental inteiramente casualizado, onde cada tratamento foi acondicionado em placas de Petri com 50 sementes, com quatro repetições, cobertas com papel celofane em diversas cores. Avaliou-se a porcentagem de germinação, índice de velocidade de germinação e o tempo médio de germinação, sendo as médias comparadas pelo teste de Tukey a 5\% de probabilidade. No ensaio, sob condições de câmara de crescimento, os tratamentos de qualidade da luz constaram de sete filtros de luz, que resultaram nas seguintes porcentagens de germinação: papel celofane incolor (luz branca) (50\%); vermelho-625 nm (36,5\%); amarelo-565nm (66\%), verde-500 nm (48,5\%), azul-440 nm (57\%), laranja- $590 \mathrm{~nm}(54 \%)$; violeta-380 nm (52\%), indicando sensibilidade das sementes à qualidade de luz.

Palavras-chaves: Hortelã., filtro de luz, planta medicinal.

\begin{abstract}
Mint spp. is an herbaceous medicinal plant, propagated by seeds or cuttings, which has great importance as an antimicrobial and anti-inflammatory in ethnobotanical and ethnopharmacological studies. This importance, due to the presence of one of its main chemical constituents, menthol, found mainly in leaves, widely used for medicinal, food and cosmetic purposes. With this, the objective was to evaluate the influence of light wave length on the germination of Mentha spp seeds. For this, a completely randomized design was used, where each treatment was placed in Petri dishes with 50 seeds, with four replications, covered with cellophane paper in different colors. The germination percentage, germination speed index and the average germination time were evaluated, with the averages compared by the Tukey test at 5\% probability. In the trial, under growth chamber conditions, the light quality treatments consisted of seven light filters, which resulted in the following germination percentages: colorless
\end{abstract}


cellophane (white light) (50\%); red-625 $\mathrm{nm}$ (36.5\%); yellow-565 nm (66\%), green-500 nm (48.5\%), blue-440 nm (57\%), orange-590 $\mathrm{nm}$ (54\%); violet-380 $\mathrm{nm}(52 \%)$, indicating seed sensitivity to light quality.

Keywords: Mint, light filter, medicinal plant.

\section{RESUMEN}

Menta spp. es una planta medicinal herbácea, propagada por semillas o esquejes, que tiene gran importancia como antimicrobiano y antiinflamatorio en estudios etnobotánicos y etnofarmacológicos. Esta importancia se debe a la presencia de uno de sus principales constituyentes químicos, el mentol, que se encuentra principalmente en las hojas, muy utilizado con fines medicinales, alimentarios y cosméticos. Con esto, el objetivo fue evaluar la influencia de la longitud de onda de la luz en la germinación de semillas de Mentha spp. Para ello, se utilizó un diseño completamente al azar, donde cada tratamiento se colocó en placas Petri con 50 semillas, con cuatro repeticiones, cubiertas con papel celofán de diferentes colores. Se evaluó el porcentaje de germinación, el índice de velocidad de germinación y el tiempo medio de germinación, con los promedios comparados por la prueba de Tukey al 5\% de probabilidad. En el ensayo, en condiciones de cámara de crecimiento, los tratamientos de calidad de la luz consistieron en siete filtros de luz, que resultaron en los siguientes porcentajes de germinación: celofán incoloro (luz blanca) (50\%); rojo-625 $\mathrm{nm}$ (36,5\%); amarillo-565 $\mathrm{nm}$ (66\%), verde-500 $\mathrm{nm}$ (48,5\%), azul-440 $\mathrm{nm}(57 \%)$, naranja-590 $\mathrm{nm}(54 \%)$; violeta-380 $\mathrm{nm}(52 \%)$, que indica sensibilidad de la semilla a la calidad de la luz.

Descriptores: Menta, filtro de luz, planta medicinal.

\section{INTRODUÇÃO}

Uma das plantas citadas como medicinal na literatura é a Mentha spp., conhecida popularmente como hortelã. Planta frequentemente citada como antimicrobiana e anti-inflamatória em estudos etnobotânicos e etnofarmacológicos. De sabor agradável e reconhecida pela RENISUS (Relação Nacional de Plantas Medicinais de Interesse ao Sistema Único de Saúde- Ministério da Saúde) é uma planta de grande interesse industrial e econômico, devido a sua produção de mentol (LORENZI e MATOS, 2008).

O estudo da fisiologia da reprodução de espécies medicinais e aromáticas é importante para garantir o aumento da população de plantas, e consequentemente o aumento da produtividade nos cultivos comerciais.

Dentro da fisiologia está o estudo da germinação, que é considerada um dos mais importantes estádios do biociclo vegetal, caracterizada por processos físicos e metabólicos de naturezas complexas que levam à retomada do crescimento do eixo embrionário, culminando com a protusão da radícula através do tegumento da semente. Estudos para as espécies com propriedades medicinais têm sido realizados demonstrando a necessidade em se definir as técnicas para obtenção de uma boa porcentagem de germinação. A água e a luz são consideradas fatores ambientais de fundamental importância no controle da germinação (MENDES e CARVALHO, 2015).

A baixa percentagem de germinação ou emergência pode ser uma consequiência de problemas como dormência das sementes, baixo vigor ou devido a fatores ambientais que por não serem bem conhecidos, dificultam o manuseio e causam prejuízos (MENEZES et al., 2004).

O processo de germinação é uma sequência de eventos fisiológicos que se inicia com a absorção de água pela semente e termina com o rompimento do tegumento e lançamento da radícula. Estudos sobre este processo contribuem para definir condições ideais de desenvolvimento. Vários fatores ambientais influenciam o processo de formação e germinação de 
sementes como: umidade, temperatura, luz, disponibilidade de nutrientes, fitohormônios inibidores e promotores com efeitos distintos sobre diferentes espécies (TAIZ e ZEIGER, 2013).

A luz é um dos fatores mais importantes para a germinação das sementes. Tanto a intensidade, o comprimento de onda quanto o fotoperíodo são variáveis conhecidas por exercerem efeito sobre a germinação de sementes que possuem dormência (COPELAND e MCDONALD, 2001).

A luz influencia o desenvolvimento vegetal de outras maneiras além da fotossíntese. Há nas células dos vegetais um pigmento de natureza protéica, denominado fitocromo, que reage ao espectro luminoso entre o azul e o vermelho intenso (TAIZ e ZEIGER, 2013).

A luz participa da ativação das sementes e está ligada ao fitocromo, que é o pigmento responsável pela captação dos sinais luminosos do ambiente (BRANCALION et al., 2012). Quando esses pigmentos absorvem luz em determinados comprimentos de ondas, mudam sua conformação, permitindo ou não a resposta fotomorfogenética (TAIZ e ZEIGER, 2013).

Este pigmento possui duas formas intercambiáveis, o fitocromo inativo (FV) e o fitocromo ativo (FVE) que regulam a germinação das sementes, floração, respostas ao fotoperiodismo, estiolamento, síntese protéica, dormência de sementes, entre outros (RAVEN et al., 2001).

$\mathrm{Na}$ luz solar são observados diferentes comprimentos de ondas; entre eles se encontram a luz vermelha (V), comprimento de onda entre 600 e 700 nm e a luz vermelho-extremo (VE), (700 a $800 \mathrm{~nm}$ ) (ZAIDAN e BARBEDO, 2004).

De acordo com o comprimento de onda no qual há germinação, as sementes são classificadas em fotoblásticas positivas, negativas ou neutras. As sementes fotoblásticas positivas precisam de luz branca ou vermelha para germinar; as fotoblásticas negativas germinam na ausência de luz ou com a luz vermelha intensa; as sementes fotoblásticas neutras germinam sob qualquer condição luminosa do espectro de luz visível (TAIZ e ZEIGER, 2013).

Neste contexto, uma das formas práticas de estudar a influência dos comprimentos de ondas de luz é através do uso de papel celofane de cores variadas, que é um polímero natural derivado da celulose. Pois devido seu aspecto de uma película fina, transparente e flexível, é capaz de absorver todas as frequências de luz e refletir somente a cor escolhida, assim é possível alterar o comprimento de onda da luz, que a semente irá receber. Porém, esse método não garante minuciosa precisão, pois este material possui alguma transparência e é possível que uma pequena quantidade de outros comprimentos de onda de luz atravesse o papel celofane, mas esse método aumenta a razão do comprimento de onda escolhido, em relação às demais. Alguns outros trabalhos também testaram a influência da intensidade luminosa utilizando folhas de papel celofone em cobertura das caixas gerbox de acordo com as cores do comprimento de ondas desejados (AZEVEDO et al., 2003; MENDES e CARVALHO, 2015; YAMASHITA et al., 2008; MENEZES et al., 2004)

Apesar de várias formas de uso e de sua importância na indústria farmacêutica, as pesquisas a respeito Mentha spp. são poucas e estão relacionadas à qualidade, produção e extração de óleos essenciais desta espécie (PEGORARO et al., 2010; SNOUSSI et al., 2015; BUSATO DE FEIRIA et al., 2016).

Diante de poucas informações em relação à germinação de sementes de hortelã e na tentativa de maximizar o processo de germinação, através da 
ampliação do conhecimento fisiológico deste processo nesta espécie, objetivou-se, avaliar a influência do comprimento de ondas de luz na germinação de sementes de Mentha spp.

\section{MATERIAL E MÉTODOS}

O experimento foi conduzido no laboratório de fitoterapia, campus de Palmas - TO, da Universidade Federal do Tocantins, no período de agosto de 2017 a julho de 2018. As sementes de Hortelã (Mentha spp.) utilizadas foram obtidas por sementes comerciais com porcentagem de pureza de $99 \%$ e germinação de $62 \%$. Para a condução dos testes de germinação, as sementes foram colocadas sobre duas folhas de papel germitest previamente umedecidas com água destilada dentro de placas de Petri cobertas com papel celofane de cores variadas.

As placas de Petri foram acondicionadas em câmaras de germinação do tipo B.O.D., equipadas com quatro lâmpadas fluorescentes brancas de 20W, fixadas internamente na porta do germinador, com temperatura de $25^{\circ} \mathrm{C}$ com variação de $+/-1^{\circ} \mathrm{C}$ e luz constante.

Os tratamentos foram constituídos por quatro repetições de 50 sementes, em delineamento inteiramente casualizado. Os tratamentos definidos da seguinte forma: $\mathrm{T} 1=$ luz branca (utilizando-se papel celofane incolor, utilizado como controle); $\mathrm{T} 2=\mathrm{luz}$ vermelha (papel celofane vermelho); T3 = luz amarelo (utilizando-se papel celofane amarelo); T4 = luz verde (utilizando-se papel celofane verde); T5= luz azul (utilizando-se papel celofane azul); T6= luz laranja (utilizando-se papel celofane laranja); T7= luz violeta (utilizando papel celofane violeta). As variáveis avaliadas foram porcentagem de germinação, índice de velocidade de germinação (IVG), e tempo médio de germinação.
Durante a condução do experimento as placas de Petri contendo as sementes e papel germitest foram irrigadas com água destilada aproximadamente a cada 24 horas. A leitura iniciou-se aos quatro dias após a semeadura, sendo consideradas sementes germinadas aquelas que apresentaram a emergência da radícula e da parte aérea, seguindo os critérios estabelecidos nas Regras de Análise de Sementes (BRASIL, 1992).

Para a determinação do índice de velocidade de germinação (IVG), a contagem das plântulas foi realizada diariamente na mesma hora, a partir do início do teste de germinação até o término do experimento, 30 dias após a semeadura (DAS), sendo as plântulas normais avaliadas e retiradas da placa de Petri e calculado pela fórmula proposta por MAGUIRE (1962).

$$
\mathrm{IVG}=\mathrm{G}_{1} / \mathrm{N}_{1}+\mathrm{G}_{2} / \mathrm{N}_{2} \ldots .+\mathrm{G}_{\mathrm{n}} / \mathrm{N}_{\mathrm{n}}
$$

onde:

IVG = Índice de velocidade de germinação;

$\mathrm{G}_{1}, \mathrm{G}_{2}, \mathrm{G}_{\mathrm{n}}=$ Número de plântulas normais computadas na primeira contagem e na última contagem;

$\mathrm{N}_{1}, \mathrm{~N}_{2}, \mathrm{~N}_{\mathrm{n}}=$ Número de dias de semeadura à primeira contagem, segunda e última contagens.

O tempo médio de germinação foi calculado segundo (LABOURIAU, 1983).

$$
\mathrm{T}=\sum \mathrm{n}_{\mathrm{i}} \times \mathrm{t}_{\mathrm{i}} / \mathrm{n}_{\mathrm{i}}
$$

onde:

$$
\begin{aligned}
& \mathrm{T}=\text { Tempo médio de germinação (dias); } \\
& \mathrm{n}_{\mathrm{i}}=\text { Número de sementes germinadas no }
\end{aligned}
$$
intervalo entre cada contagem;

$t_{i}=$ tempo médio decorrido entre o início da germinação e a i-ésima contagem. 
O índice de velocidade de germinação (IVG) foi avaliado, segundo (POPNIGIS, 1977). Os dados obtidos foram submetidos à análise de variância (ANAVA) e ao teste de Tukey a 5\% de probabilidade de erro, utilizando-se o programa computacional SISVAR (FERREIRA, 2011).

\section{RESULTADOS E DISCUSSÃO}

Na Tabela 1 estão apresentados os resultados da análise de variância. Verificou-se efeito significativo para porcentagem de germinação, índice de velocidade de germinação e tempo médio de germinação das cores nos diferentes parâmetros da germinação das sementes de Hortelã. Além disso, observou-se na análise de variância que o coeficiente de variação foi alto, podendo estar relacionado ao fato de as sementes de Hortelã serem de uma espécie domesticada, porém com alto grau de variabilidade genética que influencia também o processo de dormência e baixo vigor das sementes (MENEGHELLO et al., 2002).

Tabela 1 - Resumo da análise de variância da porcentagem de germinação (GER), índice de velocidade (IVG) e tempo médio de germinação (TMG), Palmas - TO, 2018.

\begin{tabular}{lllll}
\hline & & \multicolumn{3}{c}{ Quadrados médios } \\
\cline { 3 - 5 } Fontes de variação & GL & \% GER & IVG & TMG \\
\hline Tratamento & 6 & $321^{\text {*z }}$ & $1,2^{\text {** }}$ & $27^{\text {** }}$ \\
Erro & 21 & 131,14 & 0,21 & 3,37 \\
\hline CV $(\%)$ & 22,02 & 23,42 & 11,17 \\
\hline
\end{tabular}

ns não significativo; ${ }^{* *}$ significativo para $\mathrm{P} \leq 0,01$; ${ }^{*}$ significativo para $\mathrm{P} \leq 0,05$ pelo teste $\mathrm{F}$.

Na Tabela 2, estão apresentadas as médias referentes aos tratamentos com as diversas cores aplicadas em sementes de Hortelã, os resultados obtidos evidenciaram efeito significativo do tratamento com papel celofane amarelo (luz amarela) com maior porcentagem de germinação (66\%), diferindo estatisticamente do tratamento com papel celofane de cor vermelha e semelhante as demais cores. Observou-se também que a porcentagem de germinação de sementes de Hortelã foi baixa e variou de 36,5 a $66 \%$. A menor percentagem de germinação foi observada no tratamento com papel celofane vermelha $(36,5 \%)$.

Tabela 2 - Médias da percentagem de germinação (\%GER), índice de velocidade de germinação (IVG) e tempo médio de germinação (TMG) de sementes de Hortelã. Palmas TO, 2018.

\begin{tabular}{lccc}
\hline TRATAMENTOS & \%GER & IVG & TMG \\
\hline Branca & $50 \mathrm{ab}$ & $1,60 \mathrm{ab}$ & $17,92 \mathrm{bc}$ \\
Vermelha & $36,5 \mathrm{~b}$ & $0,92 \mathrm{~b}$ & $21,49 \mathrm{c}$ \\
Amarela & $66 \mathrm{a}$ & $2,24 \mathrm{a}$ & $16,26 \mathrm{bc}$ \\
Verde & $48,5 \mathrm{ab}$ & $2,02 \mathrm{a}$ & $15,57 \mathrm{bc}$ \\
Azul & $57 \mathrm{ab}$ & $2,56 \mathrm{a}$ & $14,10 \mathrm{bc}$ \\
Laranja & $54 \mathrm{ab}$ & $2,34 \mathrm{a}$ & $13,65 \mathrm{a}$ \\
Violeta & $52 \mathrm{ab}$ & $2,02 \mathrm{a}$ & $16,04 \mathrm{bc}$ \\
\hline
\end{tabular}

Médias seguidas pela mesma letra, na coluna não diferem significativamente entre si pelo teste de Tukey ao nível de $5 \%$ de probabilidade.

Esses resultados evidenciam a capacidade de germinação destas sementes em ambientes com luminosidade, uma vez que mesmo na simulação de vermelho (papel celofane vermelho), houve germinação, apesar de ter sido estatisticamente inferior. Isto pode estar relacionado ao fato de que neste experimento só foi usada apenas uma única folha de papel celofane, o que pode ter alterado o espectro tanto de absorção como de reflexão dos comprimentos de onda da luz que chegaram até as sementes e podem ter sido insuficientes para desencadear o processo germinativo. De acordo com a classificação de plantas fotoblástica, propostas por TAIZ e ZEIGER (2013), podemos considerar a Hortelã como fotoblástica neutra. 
A Interconversão do fitocromo pode ser o responsável pelos resultados encontrados. O fitocromo ocorre sob duas formas reversíveis, a forma fisiologicamente inativa chamada $\operatorname{Pr}$ (fitocromo vermelho), convertido durante período de luz, e a fisiologicamente ativa denominada Prf (fitocromo vermelho extremo) convertido no período de escuro e formado na luz. Portanto a fonte de luz vermelha extrema, converte o fitocromo em sua forma inativa (Pr), o que não é capaz de desencadeiar o processo germinativo (TAIZ et al., 2015).

Resultados semelhantes foram reportados por Azevedo et al. (2003), quando estudando fontes de luz na germinação de sementes de sambacaitá (Hyptis pectinata (L.)), a utilização de papel celofone amarelo revestindo as placas de Petri, propiciou uma maior porcentagem de germinação. Além disso, houve uma desuniformidade na germinação dentro de cada tratamento, sugerindo a presença de diferentes pontos de maturação das sementes na época de colheita.

Comportamento divergente foi descrito para espécies de manjericão (Ocimum basilicum L.), em experimento com diferentes intensidades de luz, em que a luz branca é mais eficiente na indução da germinação das sementes e permitiram inferir sobre seu comportamento como fotoblásticas positivas preferenciais porque, apesar de germinarem em maior porcentagem sob luz branca, também apresentaram germinação na ausência de luz (MENDES e CARVALHO, 2015).

Avaliando os efeitos da temperatura e luz na germinação e desenvolvimento de plântulas de Porophyllum ruderale não verificaram diferenças significativas na germinação tanto nas condições de luz branca como vermelho e vermelho extremo, mesmo estas variando de 12 a 44\% (YAMASHITA et al., 2008)
Ao avaliar a germinação e o crescimento de plântulas de niger (Guizotia abyssinica Cass) sob diferentes disponibilidades hídricas no substrato e regimes de luz, observaram a maior porcentagem de sementes germinadas e um maior índice de velocidade de germinação no regime de luz branca constante, permitindo-se inferir que, provavelmente, as sementes do niger são fotoblásticas negativas (ARCOVERDE et al., 2017).

A presença de luz foi favorável à germinação das sementes de sambacaitá (H. pectinata (L.)), com uma germinação média de 48\% (NETO et al., 2008). Provavelmente, estas sementes exigem a captação de comprimentos de onda de luz que é realizada por meio do fitocromo, o qual é influente na germinação (TAIZ e ZEIGER, 2013).

A semente de sálvia (Salvia splendens Sellow) comporta-se como fotoblásticas neutra em relação à luz. Quando isso ocorre, embora germine melhor na luz vermelha extrema e na ausência da luz, também, é capaz de germinar nas luzes branca e vermelha (MENEZEZ et al., 2004).

Tanto para Conyza canadensis como para $C$. bonariensis, os comprimentos de onda proporcionados pela luz verde, azul e vermelho distante reduziram a germinação das sementes, com média inferior a $5 \%$. A absorção diferencial dos comprimentos de onda do espectro da luz que chegaram até as sementes foi insuficiente para desencadear o processo germinativo. Portanto concluíram que as sementes dessas duas espécies germinam apenas na presença de luz, sendo, portanto, fotoblásticas positivas. A qualidade da luz interfere na germinação das sementes nas duas espécies, ocorrendo maior germinação sob luz branca, seguida da luz vermelha. Isso demonstra que, dependendo da qualidade de luz advinda do material usado como cobertura do solo, a germinação dessas 
espécies pode ser reduzida (YAMASHITA et al., 2011).

Resultados semelhantes foram reportados, onde a germinação de sementes de Digitaria spp. e sementes de Digitaria bicornis e Digitaria horizontalis necessitam de luz para a germinação, o que não se verifica para Digitaria ciliaris e $D$. insularis. Para essas espécies, os resultados mais favoráveis, para porcentagem de germinação, foram obtidos no regime de temperaturas alternadas $20-35^{\circ} \mathrm{C}$ e $15-35^{\circ} \mathrm{C}$, não diferentes entre si. Constataram efeito positivo da presença de luz na germinação das sementes, ou seja, há ocorrência de maior porcentagem de germinação das sementes dessa espécie, indicando característica fotoblástica positiva (MONDO et al., 2010).

A semente de Guatteria gomeziana comportou-se como uma fotoblástica neutra, uma vez que germinou tanto no escuro quanto em comprimento de ondas vermelho extremo e vermelho, sendo a germinação no escuro mais eficiente (GONÇALVES et al., 2006).

Em relação ao índice de velocidade de germinação, na Tabela 2, houve diferenças significativas entre a maioria comprimentos de luz testados. Os tratamentos realizados com as cores de papel celofane azul, laranja, verde e violeta proporcionaram os maiores índices de velocidade de germinação (2,56 a 2,02, respectivamente), diferido estatisticamente apenas dos tratamentos realizados na luz branca e no papel vermelho. Isso pode ser explicado pela opção de usar somente uma folha de papel celofane envolto nas placas, assim, a luz refletida pode ter sido menor e consequentemente, pode ter alterado os comprimentos de ondas recebidos pelas sementes, refletindo assim, no índice de velocidade de germinação. A hipótese é que o efeito da redução do comprimento de onda tenha influenciado mais os tratamentos da luz vermelha e luz branca porque, o pigmento que atua diretamente neste processo é ativado pela principalmente por estas cores, que conseguem atingir os comprimentos de ondas necessários para ativação desta molécula. Pois de acordo com Taiz e Zeiger (2013), na presença da luz vermelha ou azul, o fitocromo inativo (FV) altera sua estrutura, convertendo-se em fitocromo ativo (FVE). Ao absorver a luz vermelha intensa, o fitocromo ativo converte-se em fitocromo inativo. $\mathrm{O}$ que não ocorreu neste experimento.

De acordo com Lopes et al (2005) sob todos os comprimentos de ondas estudados, as sementes apresentaram porcentagens de germinação similares, atingindo $100 \%$ de germinação e 2,85 índice de velocidade de germinação no comprimento do vermelho, sendo, portanto, classificadas como sementes fotoblásticas neutras.

A qualidade de luz não influenciou o índice de velocidade de germinação nas temperaturas testadas, pois a velocidade de germinação parece ser mais influenciada pela temperatura. Observou-se, porém, que a luz vermelha determinou valores absolutos de índice de velocidade de germinação inferiores em estudos sobre o efeito de diferentes temperaturas e qualidades de luz sobre a germinação das sementes de sálvia (MENEZEZ et al., 2004)

Os diferentes comprimentos de luz testados não afetaram significativamente a porcentagem de germinação e o índice de velocidade de germinação, mostraram-se, inclusive, indiferentes à presença de luz, em testes avaliando os efeitos de diferentes potenciais hídricos, temperaturas e qualidade de luz na germinação de sementes de Momordica charantia L. (PARREIRA et al., 2011). 
Resultados corroboram com observações da germinação de sementes de Digitaria spp., onde verificaram que as sementes de D. bicornis e $D$. horizontalis necessitam de luz para a germinação e para elevar velocidade de germinação. Quando se comparou resultados obtidos sob iluminação com os obtidos na ausência de luz, constataram efeito positivo da presença de luz na velocidade de germinação (MONDO et al., 2010).

A porcentagem e a velocidade de germinação das sementes de Cubiu (Solanum sessiliflorum Dunal) variedade Santa Luzia acondicionadas em sacos de papel Kraft e armazenadas por seis meses em geladeira, foram superiores na condição de luz vermelha e vermelha extrema quando comparada à luz branca e à ausência de luz. Apesar de apresentar diferenças significativas entre os tratamentos, houve germinação das sementes em todas as condições de luz, atingindo valores superiores a $80 \%$ (STEFANELLO et al., 2008).

O tempo médio de germinação variou de 13,65 a 21,49 dias (Tabela 2). Os maiores tempos médios de germinação foram observados para os tratamentos com luz vermelha, luz branca, luz amarelo e luz violeta e, observa-se que o tempo médio de germinação encontrado no trabalho, está dentro dos padrões de germinação descritos nas embalagens comerciais de sementes de Hortelã que podem variar de 7 a 21 dias. Porém o menor tempo médio de germinação foi demonstrado quando foi usado o papel celofane na cor laranja, com cerca de 13 dias.

O tempo médio de germinação é uma ferramenta importante para se estimar a velocidade de ocupação de uma espécie em determinado ambiente (FERREIRA et al., 2001).

Os resultados obtidos estão em conformidade com outros, que observaram os melhores resultados de tempo médio de germinação foram dos tratamentos laranja e azul (CARVALHO e CARVALHO, 2009).

\section{CONCLUSÕES}

Nas condições em que este trabalho foi realizado, pode-se concluir que as sementes de Hortelã são capazes de germinar com luminosidade, ocorrendo preferência pela luz amarela, o que a torna como preferencial para a germinação e que os melhores resultados de índice de velocidade de germinação e tempo médio de germinação foram obtidos na presença da luz azul e laranja, respectivamente.

Todos os autores declararam não haver qualquer potencial conflito de interesses referente a este artigo.

\section{REFERÊNCIAS}

ARCOVERDE, S.N.S.; MARTINS, E.A.S.; MELO, R.M.; HARTMANN FILHO, C.P.; GORDIN, C.R.B. Germinação e crescimento de plântulas de niger sob diferentes disponibilidades hídricas do substrato e regimes de luz. Engenharia na Agricultura, v.25, n.4, p.344-353, 2017.

AZEVEDO, V.G.; SILVA-MANN, R.; COSTA, A.G.; SANTANA FILHO, L.G.M.; CARVALHO FILHO, J.L.S.; OLIVEIRA, A.S.; SANTOS, M.F.; DANTAS, I.B.; MENDONÇA, M.C.; BLANK, A.F. Influência do comprimento de onda da luz na germinação de sementes de sambacaitá [Hyptis pectinata (L.) Point]. Horticultura Brasileira. v.21, n.2, p.1-4, 2003.

BRANCALION, P.H.S.; VIANI, R.A.G.; RODRIGUES, R.R.; GANDOLFI, S. Avaliação e monitoramento de áreas em processo de restauração. In: S.V. MARTINS (Ed.), Restauração ecológica de ecossistemas degradados. Viçosa, MG: Universidade Federal de Viçosa, p. 262-293; 2012.

BRASIL. Ministério da Agricultura e da Reforma Agrária. Regras para análises de sementes. Brasília: SNDA/DNDV/CLAV, 365p. 1992.

BUSATO DE FEIRIA, S.N.; SANTANA, P.L.; BONI, G.C.; ANIBAL, P.C.; BORIOLLO, M.F.G.; FIGUEIRA, G.M.; SOUSA, I.M.O.; PEREIRA, B.; 
FOGLIO, M.A.; HÖFLING, J.F. Essential oil composition of Mentha spp. Extracted seasonally and their effects against Candida yeast growth and biofilm formation. Advancement in Medicinal Plant Research. v. 4, p. 106-115, 2016.

CARVALHO, D.B.; CARVALHO, R.I.N. Qualidade fisiológica de sementes de guanxuma sob influência do envelhecimento acelerado e da luz. Acta Scientiarum. agronomy. v.31, n.3, p.489-494, 2009.

COPELAND, L.O.; McDONALD, M.B. Principles of seed science and technology. 4 ed., Boston, MA: Kluwer Academic Publishers; 467p. 2001.

FERREIRA, A.G.; CASSOL, B.; ROSA, S.G.T.; SILVEIRA, T.S.; STIVAL, A.L.; SILVA, A.A. Germinação de sementes de Asteraceae nativas no Rio Grande do Sul, Brasil. Acta Botânica Brasílica. v.15, n.2, p.231-242, 2001.

FERREIRA, D.F. Sisvar: a computer statistical analysis system. Ciência e Agrotecnologia, v.35, n.6, p.1039-1042, 2011.

GONÇALVES, F.G.; GOMES, S.DA.S.; GUILHERME, A.L. Efeito da luz na germinação de sementes de Guatteria gomeziana (Unonopsis lindmanii R. E. FR.). Revista científica eletrônica de engenharia florestal. ANO IV, n.8, 2006.

LABOURIAU, L.G. A germinação das sementes. Washington: Secretaria Geral a Organização dos Estados Americanos, 173p. 1983.

LOPES, J.C.; CAPUCHO, M.T.; FILHO, S.M.; REPOSSI, P.A. Influência de temperatura, substrato e luz na germinação de sementes de Bertalha. Revista Brasileira de Sementes. v.27, n.2, p.18-24, 2005.

LORENZI, H.; MATTOS, F.J.A. Plantas Medicinais no Brasil - Nativas e Exóticas. 2 ed., Nova Odessa: Instituto Plantarum, 2008.

MAGUIRE, J.D. Speed of germination aid in selection and evaluation for seedling emergence and vigor. Crop Science, v.2, n.1, p.176- 177, 1962.

MENEGHELLO, G.E.; SCHNEIDER, S.M.H.; LUCCA-FILHO, O.A. Veracidade da germinação indicada nas embalagens de sementes de espécies medicinais. Revista Brasileira de Sementes, v.24, n.1, p.5-10, 2002.
MENDES, A.K.V.; CARVALHO, J.S.B. de. Germinação de sementes de manjericão em diferentes condições ambientais. Revista Ciência, tecnologia \& ambiente. v.1, n.1, p.21-27, 2015.

MENEZES, N.L.DE; FRANZIN, S.M.; ROVERSI, T.; NUNES, E.P. Germinação de sementes de Salvia splendens Sellow em diferentes temperaturas e qualidades de luz. Revista Brasileira de Sementes. v.26, n.1, p.32-37, 2004.

MONDO, V.H.V.; CARVALHO, S.J.P.DE; DIAS, A.C.R.; FILHO, J.M. Efeitos da luz e temperatura na germinação de sementes de quatro espécies de plantas daninhas do gênero Digitaria. Revista Brasileira de Sementes. v.32, n.1, p.131-137, 2010.

NETO, A.L.DOS.S.; FILHO, S.M.; TEÓFILO, E.M.; GUIMARÃES, R.M.; BLANK, A.F.; SILVAMANN, R. Influência da luz e da temperatura na germinação de sementes de sambacaitá (Hyptis pectinata (L.) Poit). Revista Brasileira Agrociência. v.14, n. 4-4, p.19-26, 2008.

PARREIRA, M.C.; CARDOZO, N.P.; GIANCOTTI, P.R.F.; ALVES, P.L.A.DA.C. Germinação de sementes de Melão-de-são-caetano sob variação de água, luz e temperatura. Bioscience Journal. v.27, n.3, p.363-370, 2011.

PEGORARO, R.L.; FALKENBERG, M.DE.B.; VOLTOLINI, C.H.; SANTOS, M.; PAULILO, M.T.S. Produção de óleos essenciais em plantas de Mentha $x$ piperita L. var. piperita (Lamiaceae) submetidas a diferentes níveis de luz e nutrição do substrato. Revista Brasileira de Botânica. v.33, n.4, p.631-637, 2010.

POPINIGS, F. Fisiologia de Sementes. Brasília: Agiplan, 289p. 1977.

RAVEN, H.P.; EVERT, R.F.; EICHHORN, S.E. Biologia vegetal. 5 ed., Rio de Janeiro, Guanabara Koogan; 2001.

SNOUSSI, M.; NOUMI, E.; TRABELSI, N.; FLAMINI, G.; PAPETTI, A.; FEO, V.DE. Mentha spicata Essential Oil: Chemical Composition, Antioxidant and Antibacterial Activities against Planktonic and Biofilm Cultures of Vibrio spp. Strains. Molecules. v.20, n.8, p.14402-14424, 2015.

STEFANELLO, S.; CHRISTOFFOLI, P.; FRANTZ, G.; ROCHA, A.C.DE.S.; SILVA, J.M.DA; STEFANELLO, R.; SCHUELTER, A.R. Germinação 
de sementes armazenadas de cubiu sob diferentes condições de luz. Scientia Agraria. v.9. n.3. p.363367, 2008.

TAIZ, L.; ZEIGER, E. Fisiologia vegetal. 5 ed., Porto Alegre; Artmed; 954p. 2013.

TAIZ, L.; ZEIGER, E.; MOLLER, I.M.; MURPHY, A. Plant physiology and development. 6 ed., Oxford University Press, Sinauer Associates; 2015.

YAMASHITA, O.M.; ALBUQUERQUE, M.C.F.E; GUIMARÃES, S.C.; SILVA, J.L.DA; CARVALHO, M.A.C.DE. Influência da temperatura e da luz na germinação de sementes de couve-cravinho
(Porophyllum ruderale (Jacq.) Cass.). Revista Brasileira de Sementes. v.30, n.3, p.202-206, 2008.

YAMASHITA, O.M.; GUIMARÃES, S.C.; CAVENAGHI, A.L. germinação das sementes de Conyza canadensis e Conyza bonariensis em função da qualidade de luz. Planta Daninha. v.29, n.4, p.737743, 2011.

ZAIDAN, L.B.P.; BARBEDO, C.J. Quebra de Dormência em Sementes. In: A. G. FERREIRA; F. BORGHETTI (orgs.), Germinação: Do Básico ao Aplicado. Porto Alegre, RS: Artmed, p.135-146; 2004. 